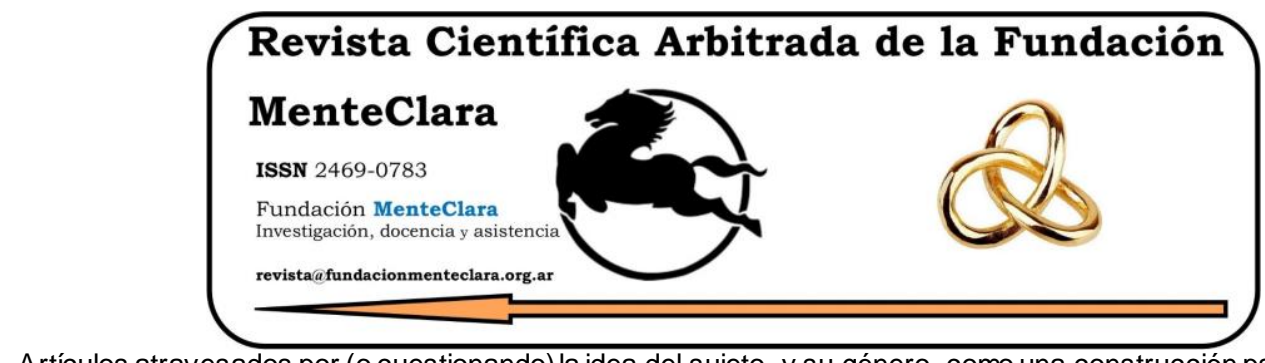

Artículos atravesados por (o cuestionando) la idea del sujeto -y su género- como una construcción psicobiológica de la cultura. Articles driven by (or questioning) the idea of the subject -and their gender- as a cultural psychobiological construction.

Vol. 7 (2022), enero-diciembre ISSN 2469-0783

https: / / datahub.io/dataset/2022-7-e266

\title{
VÍNCULO Y RELACIÓN DE OBJETO. UNA REVISIÓN BIBLIOGRÁFICA ACERCA DE LA PROPUESTA DE ENRIQUE PICHÓN-RIVIĖRE
}

BONDING AND OBJECT-ORIENTED RELATIONSHIP. A BIBLIOGRAPHIC REVIEW OF ENRIQUE PICHÓN-RIVIĖRE'S PROPOSAL

Alejandro Zarankin lic.alezarankin@gmail.com Universidad de Buenos Aires, Argentina.

Cómo citar este artículo / Citation: Zarankin, A. (2022). Vínculoy relación de objeto. Una revisión bibliográfica acerca de la propuesta de Enrique Pichón-Rivière. Revista Científica Arbitrada de la Fundación MenteClara, Vol. 7 (266). DOI: https: / / doi.org/ 10.32351/rca.v7.266

Copyright: (C) 2022 RCAFMC. Este artículo de acceso abierto es distribuido bajo los té rminos de la licencia Creative Commons Attribution 4.0 International License (CC BY 4.0). Recibido: 18/01/2022. Aceptado: 02/02/2022 Publicación online: 11/02/2022

Conflicto de intereses: Ninguno que declarar.

\section{Resumen}

El concepto de relación de objeto ha sido un potente concepto para el trabajo clínico y para la escuela de las relaciones objetales dentro del psicoanálisis desde 1930. En 1970 surge el concepto de vínculo que tiene como antecedente la relación de objeto. El objetivo de este trabajo fue identificar aquello que se mantiene y se modifica del concepto de vínculo respecto de la relación objetal. Se realizó una revisión bibliográfica de 40 artículos y textos en idioma español sobre los autores que analizaron ambos conceptos en Redalyc, SciELO, RedBUSUSAL y APAPsiBase. Se encontraron autores que abonan la postura en la que el vínculo contiene y amplía la relación de objeto. 


\begin{abstract}
The concept of object relationship has been a powerful concept for clinical work and for the school of object relations within psychoanalysis since 1930. In 1970, the concept of link arose, whose antecedent was the object relationship.

The objective of this work was to identify what is maintained and modified from the concept of link with respect to the object relationship. A bibliographic review of 40 articles and texts was carried out on the authors who analyzed both concepts in Redalyc, SciELO, RedBUSUSAL and APAPsiBase. Authors were found who support the position in which the link contains and extends the object relationship.
\end{abstract}

Palabras Claves: Vínculo; Relación de objeto/obje tal; Objeto interno; Obje to externo

Keyw ords: Bond; Bonding; object relationship; Internal object; External object 


\section{Introducción}

Entre 1932 y 1952 Klein y colaboradoras directas (Segal, Isaacs y Heimann) en Londres, Inglaterra, ayudaron a definir teóricamente el concepto princeps de la Escuela de las Relaciones Objetales: el concepto de relación de objeto o relación objetal -en lo sucesivo se hará un uso indistinto de estos dos términos-, con el acento puesto en el objeto interno (Klein, 1935/1978).

Este concepto subsume a su vez, dos conceptos: objeto interno (Klein, 1935/1978) y fantasía inconsciente (Klein, Heimann, Isaacs, \& Rivière 1943/1962). El objeto interno propone que el yo tiene una fantasía inconsciente de un ser en el interior del cuerpo que actúa hacia él. Estos objetos son introyección de personas reales, externas. La fantasía inconsciente es la representación mental de sensaciones corporales que son interpretadas como objetos internos que causan esas sensaciones. La fantasía inconsciente de relaciones con objetos internos constituye la relación objetal. Hay un objeto interno "bueno" (garante de la estabilidad del psiquismo) y un objeto interno "malo", según sean las experiencias satisfactorias o insatisfactorias respectivamente (Klein, 1957/2004).

La relación objetal como herramienta teórica, posee un alto valor para la práctica clínica. Permite poner el foco del proceso terapéutico sobre el vínculo que se establece entre paciente y analista (Tubert-Oklander, 1997). Además, tiene un valor destacado para la concepciones que abordan el desarrollo del psiquismo (Ramirez, 2010).

Sin embargo, ha sido considerado por algunos autores como limitado en su encierro en lo intrapsíquico, en la dimensión interna (Berenstein, 2001). Un concepto que lleva a un cierto solipsismo en desmedro de la realidad externa y la intersubjetividad (Moguillansky, Moreno, Ortiz 
Frágola, \& Waksman de Fisch, 1998). O bien, un concepto que incluye el mundo externo, pero no hace foco en este aspecto (Hinshelwood, 1989).

En la década de 1970 Pichón-Rivière, en Buenos Aires, Argentina, en algunas de sus definiciones, propone el concepto de vínculo (PichónRivière, 1970). Este concepto engloba una ampliación de la relación de objeto de Melanie Klein y Ronald Fairbairn (1952/1962). En este concepto, incorpora un énfasis en la interacción con la realidad (dimensión externa) sin dejar de lado el aspecto intrapsíquico (dimensión interna).

Una definición concisa del concepto de vínculo según Pichón-Rivière designa la dimensión externa de la relación de objeto internalizada. Es una estructura dinámica, en permanente transformación que contiene al sujeto y al objeto en una mutua interrelación dialéctica. La dimensión externa comprende la conducta del sujeto (Pichón-Rivière, 1970).

Respecto a los autores que abordaron previamente la articulación de ambos conceptos, a grandes rasgos hubo dos grupos. Por un lado, autores que abordaron el concepto de vínculo desde la perspectiva de psicología social de Pichón-Rivière: Pampliega de Quiroga (1978) y Santamaria (1998). Por otro, autores que lo hicieron desde la escuela de las configuraciones vinculares en psicoanálisis de Berenstein: Puget (1995), Bernard (1995) y Berenstein (1995).

Esta revisión puede ser de interés para la comunidad psicoanalítica debido a que se propuso ampliar las fronteras teóricas del concepto prínceps de la escuela de las relaciones objetales kleiniana. Esta escuela es una fecunda e importante corriente teórica dentro del Psicoanálisis.

El objetivo de la revisión fue identificar aquello que mantiene y modifica el concepto de vínculo respecto del concepto de relación objetal. 
Se produjo en el marco de la tesis de Maestría en Psicoanálisis de Alejandro Zarankin de la Universidad del Salvador (Zarankin, 2018).

\section{Materiales y métodos}

Se realizó una revisión bibliográfica de las investigaciones y textos sobre la temática del vínculo y la relación de objeto realizadas con anterioridad en español.

Se revisaron 11 artículos de investigación y 29 textos. Se utilizó para la búsqueda el sistema de información científica Redalyc que comprende la red de revistas científicas de América Latina y el Caribe, España y Portugal, la Biblioteca electrónica de ciencia y tecnología SciELO, la redBUSUSAL de la Universidad del Salvador y la Biblioteca especializada de la Asociación Psicoanalitica Argentina, a través de su base de datos virtual: APAPsiBase. Los términos de búsqueda utilizados fueron: "Vínculo y relación de objeto", "Vínculo y relación objetal".

Se analizaron los artículos y textos que abordaban estos conceptos en forma específicamente conceptual. Se excluyeron aquellos artículos y textos que se centraban en experiencias clínicas u otras temáticas. 


\section{Resultados}

Tabla 1

Resultados de búsqueda según palabras clave

\begin{tabular}{llll}
\hline Palabra clave & Buscador & Artículos & Textos \\
\hline Vínculo y relación de objeto & Redalyc & 6 & 0 \\
Vínculo y relación objetal & Redalyc & 4 & 0 \\
Vínculo y relación de objeto & SciELO & 1 & 0 \\
Vínculo y relación objetal & SciELO & 0 & 0 \\
Vínculo y relación de objeto & RedBUSUSAL & 0 & 0 \\
Vínculo y relación objetal & RedBUSUSAL & 0 & 0 \\
Vínculo y relación de objeto & APAPsiBase & 0 & 24 \\
Vínculo y relación objetal & APAPsiBase & 0 & 5 \\
\hline
\end{tabular}

Se encontraron autores que abordaron el concepto de vínculo desde la perspectiva de psicología social de Pichón-Rivière y desde la escuela de las configuraciones vinculares en psicoanálisis de Berenstein. Dentro de estos dos grupos se hallaron autores que problematizaron la articulación de ambos conceptos -relación de objeto y vínculo-, y autores que únicamente abrevaron sobre el concepto de vínculo (Puget, 1995, Friedler, 1998, Bernard, 1999, Berenstein, 2012, 2014).

La psicología social de Pichón-Rivière se sitúa entre la psicología-que estudia el psiquismo individual- y la sociología -que se ocupa de los fenómenos sociales-. La psicología social tiene su campo específico en el estudio de lo que ocurre entre los individuos. Su campo es el interrelacional (Pichón-Rivière, 1985).

Por otro lado, la escuela de las configuraciones vinculares en psicoanálisis reúne un conjunto de ámbitos terapéuticos multipersonales -psicoanálisis de familia, de pareja y de grupo-. Es el resultado de 
articular concepciones del psicoanálisis tradicional con aportes de la antropología estructural de Levi-Strauss (1958/1977).

Se encontraron 9 autores que trabajaron el vínculo en torno a la noción de intersubjetividad: Puget (1995), Berenstein (1995, 2001, 2012, 2014), Bernard (1995, 1999), Moreno, Ortiz Frágola y Waksman de Fisch (1998), Friedler (1998) y Moguillansky (1998, 1999). La intersubjetividad define al psiquismo como un sistema abierto en continuidad y discontinuidad con el otro o los otros. La comprensión del funcionamiento psíquico incluye la consideración de este sistema abierto (Spivacow, 2002).

5 autores trabajaron el vínculo como intersubjetivo que incluye lo intrasubjetivo: Pampliega de Quiroga (1978), Santamaría (1998), Bernard (2001), Vezzetti (2002) y Tubert-Oklander (2014).

7 autores trabajaron la noción de relación objetal como intrasubjetiva: Pampliega de Quiroga (1978), Berenstein (1995), Santamaría (1998), Moguillansky (1999), Bernard (2001), Vezzetti (2002) y Tubert-Oklander (2014). La intrasubjetividad, comprende el funcionamiento interno del sujeto (mundo interno). Aquí el otro, queda reducido al objeto interno (Spivacow, 2002).

\section{Discusión}

Se encontraron autores que trabajaron el vínculo como intersubjetivo. Estos autores coinciden con Berenstein, quien en su última investigación (2012) aborda el vínculo desde la perspectiva intersubjetiva y enfatiza la dimensión externa. 
También, coinciden con Moguillansky, quien en su investigación (1999), desde la perspectiva de la intersubjetividad, realizó un rastreo histórico y desarrollo conceptual de la relación de objeto y del vínculo.

Por otro lado, se encontraron otros autores que tomaron el vínculo como intersubjetivo que incluye lo intrasubjetivo. Estos autores coinciden con Vezzetti, quien desde la perspectiva intersubjetivaintrasubjetiva, en su investigación (2002), señaló la filiación kleiniana del concepto de vínculo de Pichón-Rivière. Resaltó el aporte de la teoría de los roles de Mead (1934/1972), y la teoría del campo dinámico de Lewin (1958/1988) en el concepto de vínculo. También, ponderó la dimensión externa e interna de este concepto.

\section{Conclusiones}

Un hallazgo encontrado es la constatación que hay mayor consenso en torno a la noción de relación objetal como pura o mayormente intrapsíquica, donde se privilegia la dimensión interna. Respecto de este hallazgo, es interesante resaltar la coincidencia entre la Psicología Social y la Escuela de las Configuraciones Vinculares en Psicoanálisis en considerar a la relación de objeto como intrasubjetal, más allá de las divergencias que luego presentan entre sí las diferentes escuelas, en su visión del concepto de vínculo.

Se corrobora la hipótesis de trabajo: el vínculo de Pichón-Rivière contiene y amplía el concepto de relación objetal en el sentido propuesto por Klein y colaboradoras directas. No descarta ningún elemento del concepto de relación de objeto, sólo lo amplía y complejiza agregando elementos.

Se puede pensar el vínculo de Pichón-Rivière como una ampliación de la relación objetal de Klein, colaboradoras y Fairbairn. Una extensión que 
incluye lo que la relación objetal o bien dejó de lado, o no acentuó de forma suficiente: la dimensión externa. Supone capitalizar el corpus psicoanalítico de la escuela de las relaciones objetales extendiendo sus horizontes. Desterrar la idea del encierro intrapsíquico sumando la interacción con el mundo externo. Pero a su vez, sin perder los aportes que permitió centrar la lente sobre lo interno. Esto redunda en una valorización de los autores que continuaron la línea Pichoniana para pensar el vínculo.

Se sugiere investigar las psicoterapias y a los autores que retoman los aportes del vínculo según Pichón-Rivière. 


\section{Referencias}

Aizenberg, S. (1978). Tres concepciones psicoanalíticas de Pichón-Rivière. Revista de Psicoanálisis. Asociación psicoanalítica Argentina, 35 (4), pp. 675-699.

Anzieu, D. (1986). El grupo y el inconsciente. Lo imaginario grupal. Biblioteca Nueva, Madrid, 1986.

Aulagnier, P. (1975/1977). La violencia de la interpretación. Buenos Aires: Amorrortu editores.

Berenstein, I. (1995). Vínculo e inconsciente. Apuntes para una metapsicología. Revista de la Asociación Argentina de Psicología y Psicoterapia de Grupo, 18 (1), 13-25.

Berenstein, I. (2001). El vínculo y el otro. Revista de la Asociación Argentina de Psicología y Psicoterapia de Grupo, 23 (1), 9-32.

Berenstein, I. (2012). Vínculo. Revista de Psicoanálisis, 69 (4), 1022-1025.

Berenstein, I. (2014). Diccionario de psicoanálisis argentino. (Vol 2, pp. 339-343).

Bernard, M. (1995). Inconsciente y vínculos. Revista de la Asociación Argentina de Psicología y Psicoterapia de grupo. 18 (1), 27-52.

Bernard, M. (1999). Los organizadores del vínculo. De la pulsión al otro. Revista de la Asociación Argentina de Psicología y Psicoterapia de Grupo. 22 (1), 41-70.

Bernard, M. (2001, mayo). Vínculo y Relación de objeto. II Congreso Argentino de Psicoanálisis de familia y pareja. Organizado por Asociación Argentina de Psicología y Psicoterapia de Grupo, Asociación Escuela Argentina de Psicoterapia para Graduados, Asociación Psicoanalítica Argentina y Asociación Psicoanalítica de Buenos Aires.

Brodsky, Juan, \& Duarte, Juan Pablo (2017). ¡Mamá! Posiciones, de seo y estrago. Ética y Cine Journal, 7(2),7-9.[fecha de Consulta 14 de Octubre de 2021]. ISSN: 2250-5415. Disponible en: https://www.redalyc.org/articulo.oa?id=564462745001

Cabrera Rego, Julio Oscar. (2006). El Método Clínico. Un enfoque desde la teoría del vínculo. Humanidades Médicas, 6 (3)

Campuzano, Mario (2014). MODELO VINCULAR-ESTRATÉGICO DE PSICOTERAPIA GRUPAL PSICOANALÍTICA. Vínculo - Revista do NESME, 11(1), .[fecha de Consulta 14 de Octubre de 2021]. ISSN: 1806-2490. Disponible en:

https: / / www.redalyc.org/articulo.oa?id=139439432006

Carmona Parra, Jaime Alberto, \& Gaviria Hincapié, Jorge Mario, \& Layne Bernal, Benilda (2014). Suicidio, vínculos en estudiantes universitarias colombianas. Te sis Psicológica, 9(1),102111.[fecha de Consulta 14 de Octubre de 2021]. ISSN: 1909-8391. Disponible en: https:/ / www.re dalyc.org/articulo.oa?id=139031679009

Casetta, Germán (2017). No hay Traición sin Tradición. El Cambio de Matriz Disciplinar en PichonRivière. Revista Psicologia e Saúde, 9(3),47-62.[fecha de Consulta 14 de Octubre de 2021]. ISSN: . Disponible en: https:/ /www.redalyc.org/articulo.oa?id=609864758004

Clavel de Kruyff, Fernanda (1999). Una interpretación de la interpretación psicoanalítica. Signos Filosóficos, I(1),57-69.[fecha de Consulta 16 de Octubre de 2021]. ISSN: 1665-1324. Disponible en: https://www.redalyc.org/articulo.oa?id=34300104

Dagfal, Alejandro (2015). El pasaje de la higiene mental a la salud mental en la Argentina, 19201960. El caso de Enrique Pichon-Rivière. Trashumante. Revista Americana de Historia Social, (5),10-37.[fecha de Consulta 14 de Octubre de 2021]. ISSN: 2322-9381. Disponible en: https: / / www.re dalyc.org/articulo.oa?id=455644905002 
Fairbairn, W. R. D. (1952/1962). Estudio psicoanalítico de la personalidad. Buenos Aires: Editorial Lúmen-Hormé.

Friedler, R. (1998). Diccionario de psicoanálisis de las configuraciones vinculares. (pp 451-462).

Hinshelwood, R. D. (1989). Diccionario del pensamiento Kleiniano.

Hoyos, J. J. (1985). Notas sobre el vínculo entre las relaciones de objeto y la internalización. Revista de la sociedad colombiana de psicoanálisis, 10 (1), 75-90.

Jasiner, G. (1996). Grupos centrados en la tarea: un modo de re-pensar a Enrique Pichón-Rivière. Actualidad psicológica. Asistencia y estudios psicoanalíticos argentinos (AEPA), 21 (231), pp. $27-28$.

Kaës, R. (1986/1996). "Une introduction». En Soufrance et psychopathologie des liens institués. Paris: Ed. Dunod.

Klein, M., Heimann, P., Isaacs, S., \& Rivière, J.(1943/1962 a). Naturaleza y función de la fantasía. Desarrollos en psicoanálisis (pp. 73-116). Buenos Aires: Ediciones Hormé.

Klein, M. (1935/1978). Una contribución a la psicogénesis de los estados maníaco-depresivos. Melanie Klein Obras completas. Vol. II. Contribuciones al psicoanálisis. (p. 253-278). Buenos Aires: Editorial Paidós y Hormé.

Klein, M. (1957/2004). Envidia y gratitud. Melanie Klein obras completas. Envidia y gratitud y otros trabajos. Vol. III. (pp. 181-240). Buenos Aires: Editorial Paidós.

Laplanche, J. \& Pontalis, J. B. (1967/1983). Diccionario de psicoanálisis. Madrid: Ed. Labor.

Lewin, K. (1958/1988). La teoría del campo en la ciencia social. Buenos Aires: Editorial Paidós Ibérica.

Mead, G. (1934/1972) Espíritu, persona y sociedad. Madrid: Paidós.

Mier, Raymundo (2000). La antropología ante el psicoanálisis: lasiluminaciones tangenciales. Cuicuilco, 7(18),0.[fecha de Consulta 14 de Octubre de 2021]. ISSN: 1405-7778. Disponible en: https://www.redalyc.org/articulo.oa?id=35101806

Moguillansky, R. J. (1999). Vínculo y relación de objeto. Buenos Aires: Editorial Pólemos.

Moguillansky, R., Moreno, J., Ortiz Frágola, A., \& Waksman de Fisch, F. (1998, mayo). Relación de obje to y o vínculo. Mesa Redonda organizada por la Comisión Científica de APdeBA.

Pampliega de Quiroga, Ana. (1978). El concepto de sujeto en el pensamiento de E. Pichón-Rivière. Fundamentos de una psicología definida como social. Revista de Psicoanálisis. Asociación Psicoanalitica Argenitina, 35 (3), 421-442.

Pichón-Rivière, E. (1970). Del psicoanálisis a la psicología social. Tomo I. Buenos Aires: Editorial Galerna.

Pichón-Rivière, E. (1971). Del psicoanálisis a la psicología social. Tomo II. Buenos Aires: Editorial Galerna.

Pichón-Rivière, E. (1985 a). El proceso grupal. Del psicoanálisis a la psicología social (I). Buenos Aires: Ediciones Nueva Visión.

Pichón-Rivière, E. (1985b). Teoría del vínculo. Buenos Aires: Ediciones Nueva Visión.

Pichón-Rivière, J. (2002). Diccionario de términos y conceptos de psicología y psicología social. 
Puget, J. (1995). Vinculo-relación objetal en su significado instrumental y e pistemológico. Bue nos Aires: Asociación psicoanalitica de Buenos Aires.

Puget, Janine (2006). Desvincularse como decisión -estar se parado. Vínculo - Revista do NESME, 3(3), [fecha de Consulta 14 de Octubre de 2021]. ISSN: 1806-2490. Disponible en: https:/ / www.redalyc.org/articulo.oa?id=139420856002

Ramirez, N. (2010). Las relaciones objetales y el desarrollo del psiquismo: una concepción psicoanalitica. Revista IIPSI Facultad de Psicología, 13 (2), pp. 221-230.

Resnik, S. (1998). Homenaje a Enrique Pichón-Rivière. Revista de Psicoanálisis. Asociación psicoanalítica argentina, 55 (2), pp. 287-295.

Roa Bernal \& Heimy Carolina (2012). Análisis de caso de una psicosis paranoica (caso Ela). Mental, $\mathrm{X}(18), 129-163$.[fecha de Consulta 14 de Octubre de 2021]. ISSN: 1679-4427. Disponible en: https:/ / www.re dalyc.org/articulo.oa?id=42025969008

Rodríguez Sutil, C. (2002). Objetal, intersubjetivo, vincular. El psicoanálisis anticartesiano. Intersubjetivo: Revista de Psicoterapia Psicoanalitica y Salud, 4 (2), 273-285.

SÁNCHEZ SÁNCHEZ, T. (2004). Envidia, carcoma del alma: escorzo psico-literario. Clínica y Salud, 15(1),75-95.[fecha de Consulta 14 de Octubre de 2021]. ISSN: 1130-5274. Disponible en: https:/ / www.re dalyc.org/articulo.oa?id=180617820004

Santamaria, O. (1998). Consideraciones y perspectivas acerca de la teoría del vínculo. Revista de la Sociedad colombiana de psicoanálisis, 23 (2), 271-276.

Segal, H. (1965). Introducción a la obra de Melanie Klein. Buenos Aires: Paidós.

Sicardi, A.R. F. (1979). Aportes de Enrique Pichón-Rivière a la Terapia Familiar: (un caso clínico). Terapia Familiar: Estructura, patología y terapeútica del grupo familiar, 3 (1), 48-65.

Soifer, R. (1978). Significado inconsciente del sentimiento de rabia y su relación con la patología del vínculo de amor. Revista de Psicoanálisis. Asociación psicoanalítica Argentina, 35 (4), pp. 701-734.

Spivacow, M. (2002). La perspectiva intersubjetiva y sus destinos: la terapia psicoanalitica de pareja. Aperturas psicoanaliticas, (011), http:/ / www.aperturas.org/articulo.php?articulo=205

Tabak de Bianchedi, E. (1999). De los objetos a los vínculos: descubriendo la relacionalidad / Elizabeth Tabak de Bianchedi. En Bion conocido/desconocido / Elizabeth Tabak de Bianchedi. Buenos Aires: Lugar editorial.

Tubert-Oklander, J. (1997). Proceso psicoanalitico y relaciones objetales. Disponible en la WEB: http:/ / www.aperturas.org/articulos.php?id=0000093

Tubert-Oklander, J. (2014). Diccionario de psicoanálisis argentino. (Vol 2, pp. 345-348).

Vezzetti, H. (2002). Enrique Pichón-Rivière: el vínculo y la Gestalt. Buenos Aires: X Anuario de investigaciones. Universidad de Buenos Aires. Facultad de Psicología.

Zito Lema, V. (1992). Conversaciones con Enrique Pichón-Rivière sobre el arte y la locura. ( $8^{\circ}$ ed). Buenos Aires: Ediciones Cinco.

Zito Lema, V. (2004). Homenaje a Enrique Pichón-Rivière. Docta: revista de Psicoanálisis. Asociación psicoanalítica de Córdoba, 2 (1), pp. 199-209. 